TRANSACTIONS OF THE

AMERICAN MATHEMATICAL SOCIETY

Volume 365, Number 10, October 2013, Pages 5259-5275

S 0002-9947(2013)05800-X

Article electronically published on March 25, 2013

\title{
SPECIALIZATION RESULTS IN GALOIS THEORY
}

\author{
PIERRE DÈBES AND FRANÇOIS LEGRAND
}

\begin{abstract}
The central topic of this paper is this question: is a given $k$ étale algebra $\prod_{l} E_{l} / k$ the specialization of a given $k$-cover $f: X \rightarrow B$ of the same degree at some unramified point $t_{0} \in B(k)$ ? We reduce it to finding $k$ rational points on a certain $k$-variety, which we then study over various fields $k$ of diophantine interest: finite fields, local fields, number fields, etc. We have three main applications. The first one is the following Hilbert-Grunwald statement. If $f: X \rightarrow \mathbb{P}^{1}$ is a degree $n \mathbb{Q}$-cover with monodromy group $S_{n}$ over $\overline{\mathbb{Q}}$, and finitely many suitably large primes $p$ are given with partitions $\left\{d_{p, 1}, \ldots, d_{p, s_{p}}\right\}$ of $n$, there exist infinitely many specializations of $f$ at points $t_{0} \in \mathbb{Q}$ that are degree $n$ field extensions with residue degrees $d_{p, 1}, \ldots, d_{p, s_{p}}$ at each prescribed prime $p$. The second one provides a description of the separable closure of a PAC field $k$ of characteristic $p \neq 2$ : it is generated by all elements $y$ such that $y^{m}-y \in k$ for some $m \geq 2$. The third one involves Hurwitz moduli spaces and concerns fields of definition of covers.
\end{abstract}

\section{INTRODUCTION}

If $f: X \rightarrow B$ is an algebraic cover defined over a field $k$ and $t_{0}$ is a $k$-rational point on $B$, not in the branch locus of $f$, the specialization of $f$ at $t_{0}$ is a finite $k$-étale algebra of degree $n=\operatorname{deg}(f)$ (see $\$ 2.1$ for basic terminology). For example, if $B=\mathbb{P}^{1}$ and $f$ is given by some polynomial $P(T, Y) \in k[T, Y]$, it is the product of separable extensions of $k$ that correspond to the irreducible factors of $P\left(t_{0}, Y\right)$ (for all but finitely many $t_{0} \in k$ ). The main theme of the paper is the question of whether a given $k$-étale algebra $\prod_{l} E_{l} / k$ is the specialization at some unramified point $t_{0} \in B(k)$ of a given $k$-cover $f: X \rightarrow B$ of the same degree. The classical Hilbert specialization property corresponds to the special case étale algebras are taken to be single field extensions $E / k$, and the answer is positive for at least one of them.

This question was investigated in Dèb99c and DG11 for Galois covers, and some answers are given that relate to the Regular Inverse Galois Problem and the Grunwald problem. Here we consider the situation of not necessarily Galois covers. Our approach starts with a twisting lemma which extends the twisting lemma from the previous papers and gives a general answer to our question, under some hypothesis. The answer is that there exists a certain "twisted" cover $\widetilde{g}$ : $\widetilde{Z} \rightarrow B$ such that the étale algebra is a specialization of the given cover $f$ at some point $t_{0} \in B(k)$ if there exist unramified $k$-rational points on $\widetilde{Z}$ (Lemma 2.1). The

Received by the editors September 4, 2011 and, in revised form, January 13, 2012 and January 26,2012 .

2010 Mathematics Subject Classification. Primary 11R58, 12E30, 12E25, 14G05, 14H30; Secondary $12 \mathrm{Fxx}, 14 \mathrm{Gxx}, 14 \mathrm{H} 10$.

Key words and phrases. Specialization, algebraic covers, twisting lemma, Hilbert's irreducibility theorem, Grunwald's problem, PAC fields, local fields, global fields, Hurwitz spaces. 
hypothesis is that the geometric monodromy group of the cover $f_{1}^{1}$ is the symmetric group $S_{n}$ where $n=\operatorname{deg}(f)$; it is satisfied in many practical situations. The twisting lemma is the aim of 92 In $\$ 3$ we investigate the remaining problem of finding rational points on $\widetilde{Z}$ over various fields where classical diophantine techniques can be used: PAC fields, finite fields, complete fields, number fields; see Corollaries 3.13 .3 and 3.5. Together with Lemma 2.1. these statements are our most general results on the original question.

Theorems 1-3 below, which each have their own interest, are some examples of the variety of applications of our approach. They are proved in $\$ 4$. Theorem 1 is a version of Hilbert's irreducibility theorem where a Grunwald-like conclusion is conjoined with the usual irreducibility conclusion.

Theorem 1 (Corollary 4.1). Let $f: X \rightarrow \mathbb{P}^{1}$ be a degree $n \mathbb{Q}$-cover with geometric monodromy group $S_{n}$ and $\mathcal{S}$ be a finite set of primes $p$ which are suitably large (depending on $f$ ), each given with some positive integers $d_{p, 1}, \ldots, d_{p, s_{p}}$ of sum $n$. Then $f$ has infinitely many specializations that are degree $n$ field extensions of $\mathbb{Q}$ with residue degrees $d_{p, 1}, \ldots, d_{p, s_{p}}$ at each $p \in \mathcal{S}$.

Theorem 2 is concerned with the arithmetic of PAC fields. Recall that a field $k$ is said to be PAC if every non-empty geometrically irreducible $k$-variety has a Zariski-dense set of $k$-rational points. Classical results show that in some sense PAC fields are "abundant" [FJ04, theorem 18.6.1] and a concrete example is the field $\mathbb{Q}^{\operatorname{tr}}(\sqrt{-1})$ (which is also hilbertian and whose absolute Galois group is a free profinite group of countable rank); here $\mathbb{Q}^{\text {tr }}$ denotes the field of totally real numbers (algebraic numbers such that all conjugates are real). See [FJ04 for more on PAC fields.

Theorem 2 provides explicit descriptions of the finite extensions and of the separable closure of PAC fields.

Theorem 2 (Corollary 4.3). If $k$ is a PAC field of characteristic $p$, every extension $E / k$ of degree $n$ with $p \nmid n(n-1)$ can be realized by a trinomial $Y^{n}-Y+b \in k[Y]$. Furthermore, if $p \neq 2$, the separable closure $k^{\text {sep }}$ is generated by all elements $y \in$ $k^{\text {sep }}$ such that $y^{n}-y \in k$ for some $n \geq 2$, which can be taken to be $n=[k(y): k]$ if $p=0$.

We have similar results about realizations by Morse polynomials (Corollary 4.4), and over finite fields (4.2.3).

Theorem 3 concerns Hurwitz moduli spaces of covers of $\mathbb{P}^{1}$ with fixed branch point number and fixed monodromy group. Recall that Hurwitz spaces are an important tool of the arithmetic of covers as the fields of definition of their points correspond to the fields of moduli of the covers they represent. In particular, the Regular Inverse Galois Problem over a field $k$ can be reduced to the search of $k$ rational points on them. Theorem 3 considers two cases, somewhat opposite to one another: $k$ is PAC and $k$ is a number field, and it shows that points can be found with a field of definition satisfying some more or less restrictive properties.

Let $\mathrm{H}$ be a geometrically irreducible component of some Hurwitz space defined over a field $k$ and $N$ be the degree of the definition field of the cover corresponding to the generic point of $\mathrm{H}$ over that of its branch point divisor; $N$ is also the degree of the natural cover $\mathrm{H} \rightarrow \mathrm{U}_{r}$ of the configuration space $\mathrm{U}_{r}$ for finite subsets of $\mathbb{P}^{1}$

\footnotetext{
${ }^{1}$ I.e., the Galois group of the Galois closure of $f$ over the separable closure $k^{\text {sep }}$.
} 
of cardinality $r$ (see 4.3 . We also make this assumption which can be checked in practice: the Hurwitz braid action restricted to $\mathrm{H}$ generates all of $S_{N}$ (more formally, $S_{N}$ is the geometric monodromy group of the cover $\mathrm{H} \rightarrow \mathrm{U}_{r}$ ).

Theorem 3 (Corollary 4.8). Consider the subset $\mathcal{U} \subset \cup_{r}(k)$ of all $\mathbf{t}_{0}$ such that the set $\mathrm{H}_{\mathbf{t}_{0}}$ of $\bar{k}$-covers $f: X \rightarrow \mathbb{P}^{1}$ in $\mathrm{H}$ with branch divisor $\mathbf{t}_{0}$ satisfies the following conditions (in each case):

(a) (case $k \mathrm{PAC}$ of characteristic 0$)$ : given $s$ finite extensions $E_{l} / k$ with $\sum_{l=1}^{s}\left[E_{l}\right.$ : $k]=N$, there are $s \bar{k}$-covers in $\mathrm{H}_{\mathbf{t}_{0}}$, say $f_{1}, \ldots, f_{s}$, that have smallest definition field $E_{1}, \ldots, E_{s}$ respectively, and the $N-s$ others are $k$-conjugates of $f_{1}, \ldots, f_{s}$,

(b) (case $k$ a number field):

- the moduli field of each cover $f \in \mathrm{H}_{\mathbf{t}_{0}}$ is a degree $N$ extension of $k$,

- for each $v$ in a given finite set of finite places of $k$ with suitably large residue characteristic (depending on $\mathrm{H}$ ) and for each associated partition $\left\{d_{v, 1} \ldots, d_{v, s_{v}}\right\}$ of $N$, the smallest definition fields of the covers $f \otimes_{\bar{k}} \overline{k_{v}}\left(f \in \mathrm{H}_{\mathbf{t}_{0}}\right)$ are the unramified extensions of $k_{v}$ of degree $d_{v, 1}, \ldots, d_{v, s_{v}}$.

Then (in each case) $\mathcal{U}$ is a Zariski-dense subset of $\mathbf{U}_{r}(k)$.

We refer to 4 for more detailed statements and further applications.

We wish to thank the anonymous referee for helpful comments and many valuable suggestions.

\section{The tWisting Lemma}

We first set up the terminology and notation for the basic notions we will use. The reader who is familiar with étale algebras, covers and their specializations, Galois groups, and fundamental groups and their representations can skip \$2.1 to get to the core of the paper and come back to 2.1 when needed.

2.1. Basic notation. Given a field $k$, fix an algebraic closure $\bar{k}$ and denote the separable closure of $k$ in $\bar{k}$ by $k^{\mathrm{sep}}$ and its absolute Galois group by $\mathrm{G}_{k}$. If $k^{\prime}$ is an overfield of $k$, we use the notation $\otimes_{k} k^{\prime}$ for the scalar extension from $k$ to $k^{\prime}$ : for example, if $X$ is a $k$-curve, $X \otimes_{k} k^{\prime}$ is the $k^{\prime}$-curve obtained by scalar extension. For more on this subsection, we refer to [DD97, §2] or [Dèb09, chapitre 3].

2.1.1. Étale algebras and their Galois representations. Given a field $k$, a $k$-étale algebra is a product $\prod_{l=1}^{s} E_{l} / k$ of finite sub-field extensions $E_{1} / k, \ldots, E_{s} / k$ of $k^{\mathrm{sep}} / k$. Set $m_{l}=\left[E_{l}: k\right], l=1, \ldots, s$, and $m=\sum_{l=1}^{s} m_{l}$. If $N / k$ is a Galois extension containing the Galois closures of $E_{1} / k, \ldots, E_{s} / k$, the Galois group $\operatorname{Gal}(N / k)$ acts by left multiplication on the left cosets of $\operatorname{Gal}(N / k)$ modulo $\operatorname{Gal}\left(N / E_{l}\right)$ for each $l=1, \ldots, s$. The resulting action $\operatorname{Gal}(N / k) \rightarrow S_{m}$ on the set of these $m$ left cosets, which is well defined up to equivalence (i.e. up to conjugation by an element of $\left.S_{m}\right)$, is called the Galois representation of $\prod_{l=1}^{s} E_{l} / k$ relative to $N$. Equivalently, it can be defined as the action of $\operatorname{Gal}(N / k)$ on the set of all $k$-embeddings $E_{l} \hookrightarrow N$, $l=1, \ldots, s$.

Conversely, an action $\mu: \operatorname{Gal}(N / k) \rightarrow S_{m}$ determines a $k$-étale algebra in the following way. For $i=1, \ldots, m$, denote the fixed field in $N$ of the subgroup of $\operatorname{Gal}(N / k)$ consisting of all $\tau$ such that $\mu(\tau)(i)=i$ by $E_{i}$. The product $\prod_{l} E_{l} / k$ for $l$ ranging over a set of representatives of the orbits of the action $\mu$ is a $k$-étale algebra with $\sum_{l}\left[E_{l}: k\right]=m$. If two $k$-étale algebras $\prod_{l=1}^{s} E_{l} / k$ and $\prod_{l=1}^{s^{\prime}} E_{l}^{\prime} / k$ are obtained in this manner from two different choices of the set of representatives 
of the orbits of $\mu$, then they are equivalent in the sense that $s=s^{\prime}$ and there exists $\sigma_{1}, \ldots, \sigma_{s} \in \operatorname{Gal}(N / k)$ such that $\sigma_{l}\left(E_{l}\right)=E_{l}^{\prime}, l=1, \ldots, s$. Equivalently an equivalence class of $k$-étale algebras can be viewed as a product of $k$-isomorphism classes of finite sub-field extensions of $k^{\mathrm{sep}} / k$.

G-Galois variant: if $\prod_{l=1}^{s} E_{l} / k$ is a single Galois extension $E / k$, the restriction $\operatorname{Gal}(N / k) \rightarrow \operatorname{Gal}(E / k)$ is called the $\mathrm{G}$-Galois representation of $E / k$ (relative to $N)$. Any map $\varphi: \operatorname{Gal}(N / k) \rightarrow G$ obtained by composing $\operatorname{Gal}(N / k) \rightarrow \operatorname{Gal}(E / k)$ with a monomorphism $\mathrm{Gal}(E / k) \rightarrow G$ is called a G-Galois representation of $E / k$ (relative to $N)$. The extension $E / k$ can be recovered from $\varphi: \operatorname{Gal}(N / k) \rightarrow G$ by taking the fixed field in $N$ of $\operatorname{ker}(\varphi)$. One obtains the Galois representation $\operatorname{Gal}(N / k) \rightarrow S_{n}$ of $E / k$ (relative to $N$ ) from a G-Galois representation $\varphi: \operatorname{Gal}(N / k) \rightarrow G$ (relative to $N$ ) by composing it with the left-regular representation of the image group $\varphi(\operatorname{Gal}(N / k))$; here $n=|\varphi(\operatorname{Gal}(N / k))|$.

2.1.2. Covers and function field extensions. Given a regular projective geometrically irreducible $k$-variety $B$, a $k$-mere cover of $B$ is a finite and generically unramified morphism $f: X \rightarrow B$ defined over $k$ with $X$ a normal and geometrically irreducible variety. Through the function field functor $k$-mere covers $f: X \rightarrow B$ correspond to finite separable field extensions $k(X) / k(B)$ that are regular over $k$ (i.e. $k(X) \cap \bar{k}=k)$. The Galois group of the Galois closure $\widehat{k(X)} / k(B)$ of $k(X) / k(B)$ is called the monodromy group of $f$ and the monodromy group of the $k^{\mathrm{sep}}$-mere cover $f \otimes_{k} k^{\text {sep }}$ is the geometric monodromy group. The Galois closure $\widehat{k(X)} / k(B)$ need not be a regular extension of $k$; it is if and only if the monodromy group and the geometric monodromy group coincide. This happens for example if $\widehat{k(X)}=k(X)$ (i.e. if $f$ is Galois) or if $f$ is of degree $n$ and geometric monodromy group $S_{n}$. When the Galois closure $\widehat{k(X)} / k(B)$ is regular over $k$, it corresponds to a Galois $k$-mere cover $g: Z \rightarrow B$ called the Galois closure of $f$.

The term "mere" used above is meant to distinguish mere covers from G-covers. By $k$-G-cover of $B$ of group $G$, we mean a Galois $k$-mere cover $f: X \rightarrow B$ given together with an isomorphism $G \rightarrow \operatorname{Gal}(k(X) / k(B))$. $k$-G-covers of $B$ of group $G$ correspond to regular Galois extensions $k(X) / k(B)$ given with an isomorphism of the Galois group $\operatorname{Gal}(k(X) / k(B))$ with $G$.

By a branch divisor of a $k$-cover $f$ (mere or G-), we mean that of the $k^{\text {sep }}$-cover $f \otimes_{k} k^{\text {sep }}$, i.e. the formal sum $D$ of all hypersurfaces of $B$ such that the associated discrete valuations are ramified in the extension $k^{\mathrm{sep}}(X) / k^{\mathrm{sep}}(B)$. From purity of the branch locus, $f$ is étale above $B \backslash D$.

2.1.3. $\pi_{1}$-representations. Given a reduced effective divisor $D \subset B$, denote the $k$ fundamental group of $B \backslash D$ by $\pi_{1}(B \backslash D, t)_{k}$, where $t \in B(\bar{k}) \backslash D$ is a base point. Conjoining $\S 2.1 .1$ and $\S 2.1 .2$ we obtain the following correspondences.

Mere covers of $B$ of degree $n$ (resp. G-covers of $B$ of group $G$ ) with branch divisor contained in $D$ correspond to transitive morphism $2^{2} \pi_{1}(B \backslash D, t)_{k} \rightarrow S_{n}$ such that the restriction to $\pi_{1}(B \backslash D, t)_{k}$ sep remains transitive (resp. to epimorphisms $\pi_{1}(B \backslash D, t)_{k} \rightarrow G$ such that the restriction to $\pi_{1}(B \backslash D, t)_{k}$ sep remains onto $)$. These morphisms are called fundamental group representations $\left(\pi_{1}\right.$-representations for short) of the corresponding $k$-covers (mere or G-).

\footnotetext{
${ }^{2}$ I.e. such that the image group is a transitive subgroup of $S_{n}$.
} 
2.1.4. Specializations. Each $k$-rational point $t_{0} \in B(k) \backslash D$ provides a section $\mathrm{s}_{t_{0}}$ : $\mathrm{G}_{k} \rightarrow \pi_{1}(B \backslash D, t)_{k}$ to the exact sequence

$$
1 \rightarrow \pi_{1}(B \backslash D, t)_{k^{\mathrm{sep}}} \rightarrow \pi_{1}(B \backslash D, t)_{k} \rightarrow \mathrm{G}_{k} \rightarrow 1
$$

which is uniquely defined up to conjugation by an element in the fundamental group $\pi_{1}(B \backslash D, t)_{k^{\text {sep }}}$.

If $\phi: \pi_{1}(B \backslash D, t)_{k} \rightarrow G$ represents a $k$-G-cover $f: X \rightarrow B$, the morphism $\phi \circ \mathrm{s}_{t_{0}}: \mathrm{G}_{k} \rightarrow G$ is a G-Galois representation. The kernel $\operatorname{ker}\left(\phi \circ \mathrm{s}_{t_{0}}\right)$ does not depend on the choice of $s_{t_{0}}$ up to conjugation in $\pi_{1}(B \backslash D, t)_{k}$ sep. The fixed field in $k^{\mathrm{sep}}$ of $\operatorname{ker}\left(\phi \circ \mathrm{s}_{t_{0}}\right)$ is the residue field at some point above $t_{0}$ in the extension $k(X) / k(B)$ (in fact at any point above $t_{0}$ since the extension $k(X) / k(B)$ is Galois). We denote it by $k(X)_{t_{0}}$ and call $k(X)_{t_{0}} / k$ the specialization of the $k$-G-cover $f$ at $t_{0}$.

If $\phi: \pi_{1}(B \backslash D, t)_{k} \rightarrow S_{n}$ represents a $k$-mere cover $f: X \rightarrow B$, the morphism $\phi \circ \mathrm{s}_{t_{0}}: \mathrm{G}_{k} \rightarrow S_{n}$ is the specialization representation of $f$ at $t_{0}$. The corresponding $k$-étale algebra is denoted by $\prod_{l=1}^{s} k(X)_{t_{0}, l} / k$ and called the specialization algebra of $f$ at $t_{0}$. Each field $k(X)_{t_{0}, l}$ is a residue extension at some prime above $t_{0}$ in the extension $k(X) / k(B)$ and vice-versa; $k(X)_{t_{0}, l}$ is called a specialization of $f$ at $t_{0}$. Geometrically, the fields $k(X)_{t_{0}, l}$ correspond to the definition fields of the points in the fiber $f^{-1}\left(t_{0}\right)$ and $\phi \circ \mathrm{s}_{t_{0}}: \mathrm{G}_{k} \rightarrow S_{n}$ to the action of $\mathrm{G}_{k}$ on these points. The compositum in $k^{\mathrm{sep}}$ of the Galois closures of all specializations at $t_{0}$ is the specialization at $t_{0}$ of the Galois closure of $f$.

2.2. The twisting lemma. Let $k$ be a field, $f: X \rightarrow B$ be a $k$-mere cover and $\prod_{l=1}^{s} E_{l} / k$ be a $k$-étale algebra. The question we address is whether $\prod_{l=1}^{s} E_{l} / k$ is (equivalent to) the specialization algebra $\prod_{l} k(X)_{t_{0}, l} / k$ of $f: X \rightarrow B$ at some unramified point $t_{0} \in B(k)$. Lemma 2.1 gives a sufficient condition for the answer to be affirmative.

2.2.1. Statement of the twisting lemma. We assume that $f: X \rightarrow B$ is of degree $n$ and geometric monodromy group $S_{n}$. Denote the Galois closure of the cover $f$ by $g: Z \rightarrow B$ and the compositum inside $k^{\text {sep }}$ of the Galois closures of the extensions $E_{l} / k, l=1, \ldots, s$, by $N / k$. The twisted cover $\widetilde{g}^{N}: \widetilde{Z}^{N} \rightarrow B$ in the statement below is a $k$-mere cover obtained by twisting the Galois $k$-mere cover $g: Z \rightarrow B$ by the Galois extension $N / k$. Its precise definition is given in [DG11, $\S 2.2$ ] and is recalled in 2.2 .2 It is in particular a $k$-model of $g \otimes_{k} k^{\mathrm{sep}}$; it depends on $\prod_{l=1}^{s} E_{l} / k$ only via the Galois closure $N / k$.

The next statement is a version for mere covers of the "twisting lemma" from DG11] which concerns G-covers.

Twisting lemma 2.1. Assume $f: X \rightarrow B$ is a degree $n k$-mere cover with geometric monodromy group $S_{n}$ and $\prod_{l=1}^{s} E_{l} / k$ is a $k$-étale algebra with $\sum_{l=1}^{s}\left[E_{l}\right.$ : $k]=n$. Then the twisted cover $\widetilde{g}^{N}: \widetilde{Z}^{N} \rightarrow B$ has the following property. For each unramified point $t_{0} \in B(k)$,

if (i) there exists a point $x_{0} \in \widetilde{Z}^{N}(k)$ such that $\widetilde{g}^{N}\left(x_{0}\right)=t_{0}$, then (ii) $\prod_{l} E_{l} / k$ is the specialization algebra $\prod_{l} k(X)_{t_{0}, l} / k$ of $f$ at $t_{0}$.

For $B=\mathbb{P}^{1}$, a polynomial form of the statement can be given for which the $k$-mere cover is replaced by a polynomial $P(T, Y) \in k[T, Y]$ of degree $n$ and with 
Galois group $S_{n}$ over $\bar{k}$, as a polynomial in $Y$. For all but finitely many $t_{0} \in k$, implication (i) $\Rightarrow$ (ii) holds with condition (ii) translated as follows:

(ii) the polynomial $P\left(t_{0}, Y\right)$ factors as a product $\prod_{l=1}^{s} Q_{l}(Y)$ of polynomials $Q_{l}$ irreducible in $k[Y]$ and such that $E_{l} / k$ is generated by one of its roots, $l=1, \ldots, s$.

With some adjustments, some converse (ii) $\Rightarrow$ (i) also holds in the twisting lemma 2.1. It is also possible to relax the assumption that the geometric monodromy group of $f: X \rightarrow B$ is $S_{n}$, at the cost of some technical complications. This is explained in $\mathrm{DL}$, together with some specific applications.

2.2.2. Proof of the twisting lemma. Let $H=\operatorname{Gal}(N / k), \varphi: \mathrm{G}_{k} \rightarrow H$ be the GGalois representation of $N / k$ relative to $k^{\text {sep }}$ and $\mu: H \rightarrow S_{n}$ be the Galois representation of $\prod_{l=1}^{s} E_{l} / k$ relative to $N$. The map $\mu \circ \varphi: \mathrm{G}_{k} \rightarrow S_{n}$ is then the Galois representation of $\prod_{l=1}^{s} E_{l} / k$ relative to $k^{\mathrm{sep}}$. Denote the $s$ orbits of $\mu: H \rightarrow S_{n}$, which are the same as the orbits of $\mu \circ \varphi: \mathrm{G}_{k} \rightarrow S_{n}$, by $\mathcal{O}_{1}, \ldots, \mathcal{O}_{s}$; they correspond to the extensions $E_{1}, \ldots, E_{s}$. Fix one of these orbits, i.e. $l \in\{1, \ldots, s\}$, and let $i \in\{1, \ldots, n\}$ be some index such that $E_{l}$ is the fixed field in $k^{\text {sep }}$ of the subgroup of $\mathrm{G}_{k}$ fixing $i$ via the action $\mu \circ \varphi$.

Since the $k$-mere cover $f: X \rightarrow B$ is of degree $n$ and the Galois group $\operatorname{Gal}\left(k^{\mathrm{sep}}(Z) / k^{\mathrm{sep}}(B)\right)$ is assumed to be isomorphic to $S_{n}$, the same is true of $\operatorname{Gal}(k(Z) / k(B))$. Therefore $k(Z)$ is a regular extension of $k$, or, in other words, $g: Z \rightarrow B$ is a $k$-G-cover. Let $\phi: \pi_{1}(B \backslash D, t)_{k} \rightarrow S_{n}$ be the corresponding $\pi_{1}$-representation (where $D$ is the branch divisor of $f$ ).

We will now twist the $G$-cover $g: Z \rightarrow B$ by the Galois extension $N / k$. We recall the definition of the twisted cover.

With $\operatorname{Per}\left(S_{n}\right)$, the permutation group of the set $S_{n}$, consider the map

$$
\widetilde{\phi}^{\mu \varphi}: \pi_{1}(B \backslash D, t)_{k} \rightarrow \operatorname{Per}\left(S_{n}\right)
$$

defined by this formula, where $r$ is the restriction $\pi_{1}(B \backslash D, t)_{k} \rightarrow \mathrm{G}_{k}$ : for $\theta \in$ $\pi_{1}(B \backslash D, t)_{k}$ and $x \in S_{n}$,

$$
\widetilde{\phi}^{\mu \varphi}(\theta)(x)=\phi(\theta) x(\mu \circ \varphi \circ r)(\theta)^{-1} .
$$

It is easily checked that $\widetilde{\phi}^{\mu \varphi}$ is a group homomorphism with the same restriction on $\pi_{1}(B \backslash D, t)_{k^{\text {sep }}}$ as $\phi$ (composed with the left-regular representation of $\left.S_{n}\right)$. Hence the corresponding action is transitive. Denote the corresponding $k$-mere cover by $\widetilde{g}^{N}: \widetilde{Z}^{N} \rightarrow B$ and call it the twisted cover of $g$ by the extension $N / k$; it is a $k$-model of the $k^{\text {sep }}$-mere cover $g \otimes_{k} k^{\text {sep }}$. The twisted cover $\widetilde{g}^{N}: \widetilde{Z}^{N} \rightarrow B$ was defined in DG11] (and originally in Dèb99c]), where its main property that we are using below was also given.

Let $t_{0} \in B(k) \backslash D$ and assume that condition (i) from Lemma 2.1 holds, i.e., there exists $x_{0} \in \widetilde{Z}^{N}(k)$ such that $\widetilde{g}^{N}\left(x_{0}\right)=t_{0}$. Then from [DG11, lemma 2.1], there exists $\omega \in S_{n}$ such that

$$
\phi\left(\mathrm{s}_{t_{0}}(\tau)\right)=\omega(\mu \circ \varphi)(\tau) \omega^{-1} \quad\left(\tau \in \mathrm{G}_{k}\right),
$$

where $\mathrm{s}_{t_{0}}: \mathrm{G}_{k} \rightarrow \pi_{1}(B \backslash D, t)_{k}$ is the section associated with $t_{0}$ (\$2.1.4). It follows that for $j=\omega(i)$, we have, for every $\tau \in \mathrm{G}_{k}$,

$$
\phi\left(\mathrm{s}_{t_{0}}(\tau)\right)(j)=\omega(\mu \circ \varphi)(\tau)(i),
$$

and so $j$ is fixed by $\phi\left(\mathrm{s}_{t_{0}}(\tau)\right)$ if and only if $i$ is fixed by $(\mu \circ \varphi)(\tau)$. We conclude that the specialization $k(X)_{t_{0}, j}$ and the field $E_{l}$ coincide. 


\section{VARYING THE BASE FIELD}

We consider the general problem over various base fields $k$. We start with the case of PAC fields (33.1) which, after a first result in [Dèb99c], has also been studied by Bary-Soroker; see [BS10, BS09, corollary 1.4]. \$3.2 is devoted to finite fields for which various forms of the results also exist in the literature. These two cases are presented here as special cases of our unifying approach. 3.3 and $\$ 3.4$ give newer applications to the cases where $k$ is a complete field and $k$ is a number field.

3.1. PAC fields. If $k$ is a PAC field, then condition (i) from Lemma 2.1 holds for all $t_{0}$ in a Zariski dense (but not necessarily open) subset of $B(k) \backslash D$; consequently so does condition (ii).

Corollary 3.1. Let $k$ be a PAC field and $f: X \rightarrow B$ be a $k$-mere cover of degree $n$ and geometric monodromy group $S_{n}$. If $\prod_{l=1}^{s} E_{l} / k$ is a $k$-étale algebra with $\sum_{l=1}^{s}\left[E_{l}: k\right]=n$, then for all $t_{0}$ in a Zariski dense subset of $B(k) \backslash D$, the specialization algebra $\prod_{l} k(X)_{t_{0}, l} / k$ of $f$ at $t_{0}$ is $\prod_{l} E_{l} / k$.

A similar result is $\mathrm{BS10}$, theorem 2.4]. As a special case we obtain the following statement, which is [BS09, corollary 1.4]: if $P(T, Y) \in k[T, Y]$ is a polynomial of degree $n$ and Galois group $S_{n}$ over $\bar{k}$, as a polynomial in $Y$, and if $E / k$ is a degree $n$ separable extension, then there exist infinitely many $t_{0} \in k$ such that $P\left(t_{0}, Y\right)$ is irreducible in $k[Y]$ and has a root in $\bar{k}$ that generates $E / k$.

3.2. Finite fields. Assume $k$ is the finite field $\mathbb{F}_{q}$ with $q$ elements and consider the case of covers of $\mathbb{P}^{1}$ (for simplicity). From the Lang-Weil estimates for the number of rational points on a curve over $\mathbb{F}_{q}$, condition (i) from Lemma 2.1 holds for at least one unramified point $t_{0} \in \mathbb{P}^{1}(k)$ if $q+1-2 \widetilde{\mathrm{g}} \sqrt{q}>\widetilde{r} n ! / 2$, where $\widetilde{r}$ is the branch point number of the mere cover $\widetilde{g}^{N}$ from Lemma 2.1] and $\widetilde{g}$ is the genus of its covering space $\widetilde{Z}^{N}$.

Corollary 3.2. Let $f: X \rightarrow \mathbb{P}^{1}$ be an $\mathbb{F}_{q}$-mere cover of degree $n$, with $r$ branch points and with geometric monodromy group $S_{n}$. Assume that $q \geq(r n !)^{2}$. Then for every choice of positive integers $d_{1}, \ldots, d_{s}$ (possibly repeated) such that $\sum_{l=1}^{s} d_{l}=n$, there is at least one $t_{0} \in \mathbb{F}_{q}$ such that $\prod_{l=1}^{s} \mathbb{F}_{q^{d_{l}}} / \mathbb{F}_{q}$ is the specialization algebra of $f$ at $t_{0}$.

Proof. It suffices to check that $q \geq(r n !)^{2}$ guarantees the inequality $q+1-2 \widetilde{\mathrm{g}} \sqrt{q}>$ $\widetilde{r} n ! / 2+n !$; the extra $n$ ! on the right-hand side term is here to assure that $t_{0}$ can be chosen different from $\infty$. As $\widetilde{g}^{N} \otimes_{k} k^{\text {sep }} \simeq g \otimes_{k} k^{\text {sep }}$ (where $g: Z \rightarrow \mathbb{P}^{1}$ is as before the Galois closure of $f: X \rightarrow \mathbb{P}^{1}$ ), $\widetilde{r}$ is the branch point number $r$ of $g$, which is the same as that of $f$, and $\widetilde{g}$ is the genus of $Z$.

One may assume $d=n !>1$, and consequently $r \geq 2$. The Riemann-Hurwitz formula gives $2 \widetilde{\mathrm{g}}-2=-2 d+r d-\mathcal{R}$, where $\mathcal{R}$ is the ramified point number on $Z$. It follows that $\widetilde{\mathrm{g}}=(r d / 2-1)+(2-d-\mathcal{R} / 2)<r d / 2-1$ since $2-d-\mathcal{R} / 2 \leq$ $2-d-r / 2<0$. If $\sqrt{q} \geq r d$, we then have

$$
\begin{aligned}
q+1-2 \widetilde{\mathrm{g}} \sqrt{q} \geq & r^{2} d^{2}+1-2 \widetilde{\mathrm{g}} r d \\
& >r^{2} d^{2}+1-2(r d / 2-1) r d \\
& >2 r d+1 \geq r d / 2+d .
\end{aligned}
$$


One can even evaluate the number of $t_{0} \in \mathbb{F}_{q}$ for which the conclusion holds: for example, for $s=1$ and $d_{1}=n$ it is of the form $q / n+O(\sqrt{q})$. See [DL for details on this extra conclusion (which uses the converse (ii) $\Rightarrow$ (i) in the twisting lemma alluded to in $\$ 2.2 .1$.

3.3. Complete valued fields. Assume $k$ is the quotient field of some complete discrete valuation $\operatorname{ring} A$. Denote the valuation ideal by $\mathfrak{p}$, the residue field by $\kappa$, assumed to be perfect, and its characteristic by $p \geq 0$. A $k$-étale algebra $\prod_{l=1}^{s} E_{l} / k$ is said to be unramified if each field extension $E_{l} / k$ is unramified.

Let $B$ be a smooth projective and geometrically irreducible $k$-variety given with an integral smooth projective model $\mathcal{B}$ over $A$. Let $f: X \rightarrow B$ be a degree $n k$ mere cover with branch divisor $D$. Denote the Zariski closure of $D$ in $\mathcal{B}$ by $\mathcal{D}$, the normalization of $\mathcal{B}$ in $k(X)$ by $\mathcal{F}: \mathcal{X} \rightarrow \mathcal{B}$, and its special fiber by $\mathcal{F}_{0}: \mathcal{X}_{0} \rightarrow \mathcal{B}_{0}$.

In the statement below the constant $c(f, \mathcal{B})$ only depends on $f$ and $\mathcal{B}$. It is the constant $c$ from [DG11, lemma 2.4] for $g: Z \rightarrow B$ the Galois closure of $f: X \rightarrow B$; see Remark 3.4 for more on this constant. As to condition (good-red), it assures "good reduction" of the cover as is more precisely recalled in the proof of Corollary 3.3. A more elementary characterization of it for $\mathcal{B}=\mathbb{P}_{A}^{1}$ is given at the beginning of 8.1

Corollary 3.3. Let $k, \mathcal{B}$ and $f: X \rightarrow B$ be as above and $\prod_{l=1}^{s} E_{l} / k$ be an unramified $k$-étale algebra with $\sum_{l=1}^{s}\left[E_{l}: k\right]=n$. Assume that the geometric monodromy group of $f: X \rightarrow B$ is $S_{n}$ and that these two further conditions hold:

(good-red) $p=0$ or $p>n$, each irreducible component of $\mathcal{D}$ is smooth over $A$, $\mathcal{D} \cup \mathcal{B}_{0}$ is a sum of irreducible regular divisors with normal crossings over $A$, and there is no vertical ramification ${ }^{3}$ at $\mathfrak{p}$ in the Galois closure $g: Z \rightarrow B$.

( $\kappa$-big-enough) $\kappa$ is a PAC field or is a finite field of order $q \geq c(f, \mathcal{B})$. Then there exist points $t_{0} \in B(k) \backslash D$ such that $\prod_{l=1}^{s} E_{l} / k$ is the specialization algebra of $f$ at $t_{0}$. More precisely, the set of such points $t_{0}$ contains the preimage via the map $\mathcal{B}(A) \rightarrow \mathcal{B}_{0}(\kappa)$ of a non-empty subset $F \subset \mathcal{B}_{0}(\kappa) \backslash \mathcal{D}_{0}$.

Remark 3.4. The constant $c(f, \mathcal{B})$ a priori depends on $q$ via its dependence on $\mathcal{B}$. Thus it is important to have a precise description of it; otherwise the finite field part could be vacuous (if $c(f, \mathcal{B})>q$, for example). From [DG11, addendum 2.5], for each prime $\ell \neq p$, a constant $c_{\ell}$ is given there and $c(f, \mathcal{B})$ should be larger than one of these $c_{\ell}$. For $\mathcal{B}=\mathbb{P}_{A}^{1}$, one can be quite explicit: $q \geq c(f, \mathcal{B})$ should imply $q+1-2 g \sqrt{q}>r n ! / 2$; as shown in Corollary [3.2, it suffices to take $c(f, \mathcal{B})=(r n !)^{2}$ where $r$ is the branch point number (and then the desired $t_{0}$ can even be chosen $\neq \infty)$. In the general case, the description given in [DG11, addendum 2.5] shows that $c(f, \mathcal{B})$ is "geometric" in the sense that it can be kept unchanged if $f$ is replaced by $f \otimes_{k} k^{\prime}$ for any separable base extension $k^{\prime} / k$. This allows applications for a given cover and suitably large base fields. We also recall in Addendum 3.5 that in a global situation, $c(f, \mathcal{B})$ can be chosen independent of the place. This leads to other applications for a given cover and "suitably large places" (see 4.1).

Proof. Let $\widetilde{g}^{N}: \widetilde{Z}^{N} \rightarrow B$ be the $k$-mere cover from Lemma 2.1, obtained by twisting the Galois closure $g: Z \rightarrow B$ of $f: X \rightarrow B$ by the compositum $N / k$ of the Galois

\footnotetext{
${ }^{3}$ See [DG11, \$2.3] for a precise definition of non-vertical ramification. This condition can in fact be removed here if $n \geq 3$ : according to a lemma of Beckmann Bec91, no vertical ramification may then occur (under the other assumptions $p>n$ and $\mathcal{D}$ smooth), as the geometric monodromy group $S_{n}$ is of trivial center.
} 
closures of $E_{1} / k, \ldots, E_{s} / k$. From Lemma 2.1, it suffices to show that $\widetilde{Z}^{N}$ has $k$ rational points. This (and the more precise conclusion of Corollary 3.3 ) is explained in proposition 2.2 and lemma 2.4 from [DG11, which we summarize below.

Denote by $\mathcal{G}: \mathcal{Z} \rightarrow \mathcal{B}$ the normalization of $\mathcal{B}$ in $k(Z)$. Assumption (good-red) holds for $\mathcal{G}$ as it holds for $\mathcal{F}$ ( $f$ and $g$ have the same branch divisor), and the Galois extension $N / k$ is unramified (compositum of unramified extensions). These two conditions guarantee that the morphism $\widetilde{\mathcal{G}}^{N}: \widetilde{\mathcal{Z}}^{N} \rightarrow \mathcal{B}$ obtained by normalizing $\mathcal{B}$ in $k\left(\widetilde{Z}^{N}\right)$ has good reduction [DG11, proposition 2.2]; more precisely, the proof of that result shows that $\widetilde{\mathcal{G}}^{N}$ is flat, étale above $\mathcal{B} \backslash \mathcal{D}$, and the special fiber $\widetilde{\mathcal{Z}}_{0}^{N}$ is normal and geometrically irreducible [DG11, §2.4.1-4]. Assumption ( $\kappa$-big-enough) shows next that $\kappa$-rational points exist on the special fiber $\widetilde{\mathcal{Z}}_{0}^{N}$ [DG11, §2.4.5]; if $\kappa$ is finite, this follows from the Lang-Weil estimates [DG11, lemma 2.4]. Hensel's lemma is finally used to lift these $\kappa$-rational points to $k$-rational points on $\widetilde{Z}^{N}$.

3.4. Local-global results. Finding rational points on varieties over a global field $k$ is harder than it is over local fields. Nevertheless, results from 33.3 can be used to obtain local-global statements. We explain below how to globalize local information coming from Corollary 3.3 .

Let $k$ be the quotient field of some Dedekind domain $R$ and $\mathcal{S}$ be a finite set of places of $k$ corresponding to some prime ideals in $R$. For every place $v$, the completion of $k$ is denoted by $k_{v}$, the valuation ring by $R_{v}$, the valuation ideal by $\mathfrak{p}_{v}$, the residue field by $\kappa_{v}$ which we assume to be perfect, the order (possibly infinite) of $\kappa_{v}$ by $q_{v}$ and its characteristic by $p_{v}$.

Let $B$ be a smooth projective and geometrically integral $k$-variety, given with an integral model $\mathcal{B}$ over $R$ such that $\mathcal{B}_{v}=\mathcal{B} \otimes_{R} R_{v}$ is smooth for each $v \in \mathcal{S}$. The weak approximation property below guarantees that $k_{v}$-rational points on $B(v \in \mathcal{S})$ that may be provided by Corollary 3.3 can be approximated by some $k$-rational point on $B$.

(weak-approx $/ \mathcal{S}) \quad B(k)$ is dense in $\prod_{v \in \mathcal{S}} B\left(k_{v}\right)$.

The next statement then readily follows from Corollary 3.3

Corollary 3.5. Let $k, \mathcal{S}, \mathcal{B}$ be as above, $f: X \rightarrow B$ be a degree $n k$-mere cover with branch divisor $D$, and, for each $v \in \mathcal{S}$, let $\prod_{l=1}^{s_{v}} E_{v, l} / k_{v}$ be an unramified $k_{v}$-étale algebra with $\sum_{l=1}^{s_{v}}\left[E_{v, l}: k_{v}\right]=n$.

Assume that

- the geometric monodromy group of $f: X \rightarrow B$ is $S_{n}$,

- the weak approximation condition (weak-approx $/ \mathcal{S}$ ) holds, and

- for each $v \in \mathcal{S}$, conditions (good-red) and ( $\kappa$-big-enough) of Corollary 3.3 hold for the $k_{v}$-mere cover $f_{v}=f \otimes_{k} k_{v}$ and the residue field $\kappa_{v}$.

Then there exist $v$-adic open subsets $U_{v} \subset B\left(k_{v}\right) \backslash D(v \in \mathcal{S})$ such that $B(k) \cap$ $\prod_{v \in \mathcal{S}} U_{v} \neq \emptyset$ and the following holds: for each $t_{0} \in B(k) \cap \prod_{v \in \mathcal{S}} U_{v}$ and each $v \in \mathcal{S}$, the étale algebra $\prod_{l=1}^{s_{v}} E_{v, l} / k_{v}$ is the specialization algebra of $f \otimes_{k} k_{v}$ at $t_{0}$.

Addendum 3.5. Each condition $q_{v} \geq c\left(f_{v}, \mathcal{B}_{v}\right)(v \in \mathcal{S})$ from assumption $\left(\kappa_{v}\right.$-bigenough) can be guaranteed by some condition $q_{v} \geq C(f, \mathcal{B})$ where $C(f, \mathcal{B})$ only depends on $f$ and $\mathcal{B}$ (and not on $v$ ); see [DG11, lemma 3.1]. The constant $C(f, \mathcal{B})$ here is the constant $C(g, \mathcal{B})$ from there with $g$ the Galois closure of $f$. For $\mathcal{B}=\mathbb{P}_{R}^{1}$, it can be taken to be $C(f, \mathcal{B})=(r n !)^{2}$, where $r$ is the branch point number of $f$. 


\section{Applications}

The three subsections of this section correspond to the three main applications presented in the introduction.

4.1. Hilbert's irreducibility theorem. We elaborate on the local-global results from 3.4 when $B=\mathbb{P}^{1}$. In this situation, assumption (weak-approx $/ \mathcal{S}$ ) holds for every $\mathcal{S}$ (from the Artin-Whaples approximation theorem), and the good reduction assumption (good-red) requires that each place $v \in \mathcal{S}$ be good, by which we mean that $p_{v}=0$ or $p_{v}>n$, the branch point set $\mathbf{t}=\left\{t_{1}, \ldots, t_{r}\right\}$ of $f$ is étale (i.e., no two distinct branch points coalesce modulo the valuation ideal of $v$ ) and there is no vertical ramification in the Galois closure of $f 4$

4.1.1. A standard trick. Over certain fields (e.g. number fields), there is a trick that makes it possible, at the cost of throwing in more places in $\mathcal{S}$, to further guarantee in Corollary 3.5 that Hilbert's irreducibility conclusion holds, i.e. that the specialization algebra of $f$ at $t_{0}$ consists of a single field extension $k(X)_{t_{0}} / k$ of degree $n$.

Namely, the idea is to construct a finite set $\mathcal{S}_{0}$ of finite places of $k$, disjoint from $\mathcal{S}$, and to attach to each $v \in \mathcal{S}_{0}$ a $k_{v}$-étale algebra $\prod_{l} E_{v, l} / k_{v}$ with all $E_{v, l} / k_{v}$ trivial but one consisting of an unramified cyclic extension $E_{v} / k_{v}$ of degree $d_{v} \leq n$. If the assumptions of Corollary 3.5] still hold for the set $T=\mathcal{S} \cup \mathcal{S}_{0}$, then it follows from its conclusion that the Galois group $\operatorname{Gal}\left(k(Z)_{t_{0}} / k\right.$ ) (of the specialization of the Galois closure of $f$ at $\left.t_{0}\right)$ contains some cycle of length $d_{v}\left(v \in \mathcal{S}_{0}\right)$. This implies that $\operatorname{Gal}\left(k(Z)_{t_{0}} / k\right)$ is all of $S_{n}$ if for example $\mathcal{S}_{0}$ contains 3 places with the corresponding degrees $d_{v}$ equal to $2, n-1$ and $n$. Of course, for this idea to work, cyclic extensions $E_{v} / k_{v}$ of order $d_{v}$ should exist for places $v$ satisfying the assumptions of Corollary 3.5 .

4.1.2. The number field case. We develop the number field case for which this trick can be used. Another example would be to work over $k=\kappa(x)$ with $\kappa$ a PAC field with enough cyclic extensions. We will also use the explicit aspect of [DG11] that makes it possible to be more precise on the constants. For simplicity take $k=\mathbb{Q}$.

Corollary 4.1. Let $f: X \rightarrow \mathbb{P}^{1}$ be a degree $n \mathbb{Q}$-mere cover with geometric monodromy group $S_{n}$. There exist integers $m_{0}, \beta>0$ depending on $f$ with the following property. Let $\mathcal{S}$ be a finite set of good primes $p>m_{0}$, each given with positive integers $d_{p, 1}, \ldots, d_{p, s_{p}}$ (possibly repeated) with $\sum_{l=1}^{s_{p}} d_{p, l}=n$. Then there exists $b \in \mathbb{Z}$ such that

$\left(^{*}\right)$ for each integer $t_{0} \equiv b \bmod \left(\beta \prod_{p \in \mathcal{S}} p\right), t_{0}$ is not a branch point of $f$ and the specialization algebra of $f$ at $t_{0}$ consists of a single field extension $\mathbb{Q}(X)_{t_{0}} / \mathbb{Q}$ of degree $n$ which has residue degrees $d_{p, 1}, \ldots, d_{p, s_{p}}$ at $p$ for each $p \in \mathcal{S}$ and has $S_{n}$ as a Galois group of its Galois closure.

Addendum 4.1 (on the constants). Denote the number of branch points of $f$ by $r$ and the number of bad primes by $\operatorname{br}(\mathbf{t})$. One can take $m_{0}$ such that the interval $\left[(r n !)^{2}, m_{0}\right]$ contains at least $\operatorname{br}(\mathbf{t})+3$ distinct primes and $\beta$ is the product of 3 good primes in $\left[(r n !)^{2}, m_{0}\right]$.

If the cover $f: X \rightarrow \mathbb{P}^{1}$ is given by a polynomial $P(T, Y) \in \mathbb{Q}[T, Y]$, Addendum 4.1 provides a bound for the least specialization $t \geq 0$ making $P(t, Y)$ irreducible

\footnotetext{
${ }^{4}$ This last condition is automatic if the geometric monodromy group is $S_{n}$ with $n=\operatorname{deg}(f) \geq 3$.
} 
in $\mathbb{Q}[Y]$ that depends only on $\operatorname{deg}_{Y}(P), r$ and $\operatorname{br}(\mathbf{t})$. It is conjectured that a bound depending only on $\operatorname{deg}(P)$ exists in general for Hilbert's irreducibility theorem (see [DW08].

Proof. Take $m_{0}$ as in Addendum 4.1. Then $m_{0} \geq(r n !)^{2}=C\left(f, \mathbb{P}_{\mathbb{Z}}^{1}\right)$ (Addendum 3.5). Furthermore, 3 good primes can be picked in the interval $\left[(r n !)^{2}, m_{0}\right]$. Given an integer $d \geq 1$, denote the unramified extension of $\mathbb{Q}_{p}$ of degree $d$ by $E_{p}^{\mathrm{ur}, d} / \mathbb{Q}_{p}$. For $p \in \mathcal{S}$, consider the $\mathbb{Q}_{p}$-étale algebra $\underline{E}_{p}=\prod_{l=1}^{s_{p}} E_{p}^{\mathrm{ur}, d_{p, l}} / \mathbb{Q}_{p}$. Denote the set of additional primes by $\mathcal{S}_{0}=\left\{\pi_{2}, \pi_{n-1}, \pi_{n}\right\}$, and for $i=2, n-1, n$, let $\underline{E}_{\pi_{i}}=$ $\prod_{l} E_{\pi_{i}, l} / \mathbb{Q}_{\pi_{i}}$ be the $\mathbb{Q}_{\pi_{i}}$-étale algebra with one term $E_{\pi_{i}, l} / \mathbb{Q}_{\pi_{i}}$ equal to $E_{\pi_{i}}^{\mathrm{ur}, i} / \mathbb{Q}_{\pi_{i}}$ and all $n-i$ others trivial.

Apply Corollary 3.5 to the cover $f$, the set of places $\mathcal{S} \cup \mathcal{S}_{0}$ and the associated $\mathbb{Q}_{p}$-algebras $\underline{E}_{p}$. Let $t_{0}$ be in the set $\mathbb{P}^{1}(\mathbb{Q}) \cap \prod_{p \in \mathcal{S} \cup \mathcal{S}_{0}} U_{p}$ provided by its conclusion. As recalled in \$4.1.1 the 3 primes in $\mathcal{S}_{0}$ guarantee that the specialization of the Galois closure of $f$ at $t_{0}$ has Galois group $S_{n}$. In particular, the specialization of $f$ at $t_{0}$ is a single field extension $\mathbb{Q}(X)_{t_{0}} / \mathbb{Q}$ of degree $n$ (and with $S_{n}$ as Galois group of its Galois closure). The conclusion of Corollary 3.5 relative to the places in $\mathcal{S}$ yields that the field extension $\mathbb{Q}(X)_{t_{0}} / \mathbb{Q}$ has residue degrees $d_{p, 1}, \ldots, d_{p, s_{p}}$ at $p$ for each $p \in \mathcal{S}$.

In order to obtain that $t_{0}$ can be chosen to be any term of the arithmetic progression from statement $(*)$, we use the more precise description of the $p$-adic open subsets $U_{p}$ given in Corollary 3.3. for each $p \in \mathcal{S} \cup \mathcal{S}_{0}, U_{p}$ contains the preimage via the map $\mathbb{P}_{\mathbb{Z}_{p}}^{1} \rightarrow \mathbb{P}_{\mathbb{F}_{p}}^{1}$ of a non-empty subset of $\mathbb{P}_{\mathbb{F}_{p}}^{1}$, which can further be assumed to be contained in $\mathbb{A}_{\mathbb{F}_{p}}^{1}$. The weak approximation theorem then reduces to the Chinese remainder theorem and provides the desired conclusion.

4.2. Trinomial realizations and variants. Bary-Soroker's motivation in BS09] was to obtain analogs of Dirichlet's theorem for polynomial rings. He proved that if $k$ is a PAC field, then given $a(Y), b(Y) \in k[Y]$ relatively prime, for every integer $n$, suitably large (depending on $a(Y), b(Y)$ ) and for which $k$ has at least one degree $n$ separable extension, there are infinitely many $c(Y) \in k[Y]$ such that $a(Y)+$ $b(Y) c(Y)$ is irreducible in $k[Y]$ and of degree $n$. A first stage is to construct $c_{0}(Y) \in$ $k[Y]$ such that $a(Y)+b(Y) c_{0}(Y) T \in k[T, Y]$ is absolutely irreducible, of degree $n$ and Galois group $S_{n}$ over $\bar{k}(T)$. Using results as in 3.1 one can then specialize $T$ in $k$ to obtain the desired polynomials.

Below we develop other applications.

4.2.1. Classical regular realizations of $S_{n}$. A hypothesis in our results from 93 is that the $k$-mere cover $f: X \rightarrow B$ is of degree $n$ and of geometric monodromy group $S_{n}$. Below we recall some classical covers $f: X \rightarrow \mathbb{P}^{1}$ with these properties. The cover $f$ is given by a polynomial $P(T, Y) \in k[T, Y]$, the map $f$ corresponding to the $T$-projection $(t, y) \rightarrow t$ from the curve $P(t, y)=0$ to the line. Fix the integer $n \geq 2$.

(a) (Trinomials): $k$ is a field and $P(T, Y)=Y^{n}-T^{r} Y^{m}+T^{s} \in k[T, Y]$, where $n, m, r, s$ are positive integers such that $1 \leq m<n,(m, n)=1$, the characteristic $p \geq 0$ of $k$ does not divide $m n(m-n)$ and $s(n-m)-r n=1$. The branch points of the associated cover $f: X \rightarrow \mathbb{P}^{1}$ are $0, \infty$ and $t_{0}=m^{m} n^{-n}(n-m)^{n-m}$ with corresponding ramification indices $m(n-m)$ at $0, n$ at $\infty$ and 2 at $t_{0}$. See Sch00, $\S 2.4]$. 
There are other classical trinomials realizing $S_{n}$; see [Ser92, §4.4]:

- $P(T, Y)=Y^{n}-Y^{n-1}-T$ for $p$ not dividing $n(n-1)$, which has branch points $0, \infty, Q(1-(1 / n))$ with $Q(Y)=Y^{n}-Y^{n-1}$, and ramification indices $n$ at $\infty, n-1$ at 0 and 2 at $Q(1-(1 / n))$,

- $P(T, Y)=Y^{n}-Y-T$ for $p$ not dividing $n(n-1)$; this last example is a special case of (b) below.

(b) (Morse polynomials): $k$ is of characteristic $p \geq 0$ not dividing $n$ and $P(T, Y)$ $=M(Y)-T$, where $M(Y) \in k[Y]$ is a degree $n$ Morse polynomial. That is, the zeroes $\beta_{1}, \ldots, \beta_{n-1}$ of the derivative $M^{\prime}$ are simple and $M\left(\beta_{i}\right) \neq M\left(\beta_{j}\right)$ for $i \neq j$. The branch points of the cover $f: X \rightarrow \mathbb{P}^{1}$ are $\infty$ and $M\left(\beta_{1}\right), \ldots, M\left(\beta_{n-1}\right)$, with ramification indices $n$ at $\infty$ and 2 at $M\left(\beta_{1}\right), \ldots, M\left(\beta_{n-1}\right)$. See [Ser92, §4.4].

(c) (An example of Uchida): Let $k$ be any field and $U_{0}, \ldots, U_{3}$ be 4 algebraically independent indeterminates. It is proved in Uch70, corollary 2] that for every $n \geq 4$, the polynomial $F(Y)=Y^{n}+U_{3} Y^{3}+U_{2} Y^{2}+U_{1} Y+U_{0}$ has Galois group $S_{n}$ over the field $k\left(U_{0}, \ldots, U_{3}\right)$. The following lemma makes it possible to derive a polynomial

$$
P(T, Y)=Y^{n}+u_{3}(T) Y^{3}+u_{2}(T) Y^{2}+u_{1}(T) Y+u_{0}(T) \in k[T, Y]
$$

of Galois group $S_{n}$ over $\bar{k}(T)$.

Lemma 4.2. Let $\underline{U}=\left(U_{1}, \ldots, U_{\ell}\right)$ be a set of algebraically independent indeterminates and $F(\underline{U}, Y) \in k(\underline{U})[Y]$ be a degree $n$ polynomial with Galois group $S_{n}$ over $\bar{k}(\underline{U})$. Then there exist infinitely many $\ell$-tuples $\underline{u}(T)=\left(u_{1}(T), \ldots, u_{\ell}(T)\right) \in k[T]^{\ell}$ such that the polynomial $F(\underline{u}(T), Y)$ has Galois group $S_{n}$ over $\bar{k}(T)$.

Proof. The polynomial $F(\underline{U}, Y)$ has Galois group $S_{n}$ over the field $\bar{k}(T)(\underline{U})$. The desired conclusion follows from the Hilbert specialization property of the hilbertian field $\bar{k}(T)$, but one needs a version that provides good specializations in $k(T)$ (but still good relative to irreducibility over $\bar{k}(T)$ ). This is classical if $k$ is infinite (e.g. [FJ04, §13.2]). For the general case, we resort to theorem 3.3 from Dèb99b] which shows that given a Hilbert subset $\mathcal{H} \subset \bar{k}(T)$, for all but finitely many $t_{0} \in \bar{k}(T)$, there exists $a \in \bar{k}(T)$ such that if $b \in k[T]$ is any non-constant polynomial, then $\mathcal{H}$ contains infinitely many elements of the form $t_{0}+a b^{m}(m \geq 0)$. This gives what we want if $a$ can be chosen in $k(T)$. Although this is not stated, the proof shows that such a choice is possible. The main point is to adjust [Dèb99b, lemma 3.2] to show there are infinitely many cosets of $k(T)$ modulo $\bar{k}(T)^{p}$, where $p$ is the characteristic of $k$.

4.2.2. Special realizations of extensions of PAC fields. We say a field extension $E / k$ can be realized by a polynomial $Q(Y) \in k[Y]$ if $Q(Y)$ is the irreducible polynomial over $k$ of some primitive element of $E / k$.

Corollary 4.3. Let $k$ be a PAC field of characteristic $p \geq 0$. If $n \geq 2$ and $p$ does not divide $n(n-1)$, every degree $n$ extension $E / k$ can be realized by a trinomial $Y^{n}-Y+b$ for some $b \in k$. Furthermore, if $p \neq 2$, the separable closure $k^{\text {sep }}$ is generated over $k$ by all elements $y \in k^{\text {sep }}$ such that $y^{n}-y \in k$ for some integer $n \geq 2$, which can be taken to be $n=[k(y): k]$ if $p=0$.

Proof of Corollary 4.3. The first part follows from Corollary 3.1 applied with $f$ : $X \rightarrow \mathbb{P}^{1}$ given by the trinomial $P(T, Y)=Y^{n}-Y-T$ from 4.2 .1 (a) and the étale 
algebra $\prod_{l=1}^{s} E_{l} / k$ taken to be the field extension $E / k$. To prove the second part, consider a separable extension $E / k$ of degree $m \geq 2$. Pick an integer $n \geq m$ such that $p$ does not divide $n(n-1)$ (this is possible as $p \neq 2$, and one can take $n=m$ if $p=0)$, and do as above but with the étale algebra $\prod_{l=1}^{s} E_{l} / k$ taken to be the product of the field extension $E / k$ with $n-m$ copies of the trivial extension $k / k$. Conclude that $E / k$ has a primitive element whose irreducible polynomial divides $Y^{n}-Y+b$ (and is equal to $Y^{n}-Y+b$ if $p=0$ and $n=m$ ) for some $b \in k$. As $E / k$ is an arbitrary finite extension, this provides the announced description of $k^{\text {sep }}$.

Proceeding as above but using the Morse polynomial realization (b) from \$4.2.1 (instead of trinomial realizations), we obtain the following statement.

Corollary 4.4. Let $n \geq 2$ be an integer, $k$ be a PAC field of characteristic $p \geq 0$ not dividing $n$ and $M(Y) \in k[Y]$ be a degree $n$ Morse polynomial. Then every degree $n$ extension $E / k$ can be realized by a polynomial $M(Y)+b$ for some $b \in k$.

Finally, Uchida's example and Lemma 4.2 from 4.2 .1 (c) yield this.

Corollary 4.5. Let $n \geq 4$ be an integer and $k$ be a PAC field of any characteristic. Then every separable degree $n$ extension $E / k$ can be realized by a polynomial $Y^{n}+$ $a Y^{3}+b Y^{2}+c Y+d$ for some $a, b, c, d \in k$.

4.2.3. Variants. (a) Finite fields. Proceeding as above but using Corollary 3.2 instead of Corollary 3.1 leads to the following conclusions for finite fields:

- if $n \geq 2$ and $q \geq(n n !)^{2}$ is a prime power with $(q, n(n-1))=1$, the extension $\mathbb{F}_{q^{n}} / \mathbb{F}_{q}$ can be realized by a trinomial $Y^{n}-Y+b \in \mathbb{F}_{q}[Y]$,

- if $M(Y) \in \mathbb{F}_{q}[Y]$ is a degree $n$ Morse polynomial such that $(n, q)=1$ and $q \geq$ $(n n !)^{2}$, the extension $\mathbb{F}_{q^{n}} / \mathbb{F}_{q}$ can be realized by the polynomial $M(Y)+b$ for some $b \in \mathbb{F}_{q}$.

(b) $p$-adic fields. It follows from (a) that

- if $p \geq(n n !)^{2}$ is a prime, the degree $n$ unramified extension of $\mathbb{Q}_{p}$ can be realized by a trinomial $Y^{n}-Y+b$ for some $b \in \mathbb{Z}_{p}$ or by a polynomial $M(Y)+b$ with $b \in \mathbb{Z}_{p}$ and $M(Y) \in \mathbb{Z}_{p}[Y]$ a degree $n$ monic polynomial with reduction modulo $p$ a Morse polynomial in $\mathbb{F}_{p}[Y]$.

This can also be proved by using $₫ 3.3$ instead of $₫ 3.2$ (with possibly another bound on $p$ ).

(c) Other trinomials. The trinomials $Y^{n}-Y^{n-1}-T$ and $Y^{n}-T^{r} Y^{m}+T^{s}$ from 4.2 .1 can be used instead of $Y^{n}-Y-T$ to provide similar conclusions. The assumption on $p$ remains that $p \nmid n(n-1)$ for the former, and for the latter, it is that $p \nmid m n(n-m)$ (with the other conditions on $n$ and $m$ from \$4.2.1); the bound on $q$ can be replaced by the better one $q=p^{f} \geq(3 n !)^{2}$.

(d) Missing characteristics. Given an integer $n \geq 2$ and a prime $p$, Corollary 3.1. combined with Lemma 4.2, shows in fact that

$\left(^{*}\right)$ every degree $n$ separable extension $E / k$ of a PAC field $k$ of characteristic $p$ can be realized by some trinomial $Y^{n}+a Y^{m}+b$ for some integer $1 \leq m<n$ and some $a, b \in k$,

provided that the following holds:

(**) there exists $1 \leq m<n$ such that the trinomial $Y^{n}+U Y^{m}+V$ has Galois group $S_{n}$ over $\overline{\mathbb{F}}_{p}(U, V)$ (where $U, V$ are two indeterminates). 
There are many results about condition $\left(^{* *}\right)$ in the literature, notably in the papers Uch70, Coh80, and Coh81. Here are conclusions that can be derived about the cases not covered by Corollary 4.3 .

- if $p \neq 2, p \mid n(n-1)$ and $n$ is odd, (**) holds with $Y^{n}+U Y^{2}+V$ or with $Y^{n}-U Y+V$ (Coh81, corollary 3] and [Uch70, theorem2]),

- if $p=2$ and $n$ is odd, (**) holds with $Y^{n}+U Y^{2}+V$ if $n \geq 5$ Coh81, corollary $3]$ and with $Y^{n}-U Y+V$ if $n=3$ [Uch70, theorem2],

- if $p=3$ and $n=4,(* *)$ holds with $Y^{n}-U Y+V$ [Ch70, theorem2],

- if $(p=5$ and $n=6)$ or $(p=2$ and $n=6),\left({ }^{*}\right)$ does not hold: $Y^{6}-U Y+V$ has Galois group $\mathrm{PGL}_{2}\left(\mathbb{F}_{5}\right)$ over $\mathbb{F}_{5}(U, V)$ and $Y^{6}-U Y+V$ has Galois group $A_{5}$ over $\mathbb{F}_{4}(U, V)$ Uch70]. (Note that the exponent $m$ is necessarily prime to $n$ if $\left(^{* *}\right)$ holds (otherwise the Galois group of the trinomial is not primitive) and that changing $Y$ to $1 / Y$ reduces the check of $(* *)$ to half of the remaining $m$.)

Remark 4.6. From above, condition (**) holds if $n$ is odd and $p \mid n(n-1)$, and from Uch70, theorem 1] it also holds if $p \nmid n(n-1)$ (with $m=1$ ). Hence condition $\left({ }^{* *}\right)$, and so condition $(*)$ too, always holds if $n$ is odd.

(e) Number fields. Over a number field $k$, extensions with trinomial realizations are more sparse. For example, Angeli proved that, for every $n \geq 3$, there are (up to some standard equivalence for trinomials) only finitely many degree $n$ trinomials with coefficients in $k$, irreducible and with Galois group a primitive subgroup $G \subset$ $S_{n}$ distinct from $S_{n}$ and $A_{n}$ Ang09. See also Ang07 where the same is proved with " $G \subset S_{n}$ primitive" replaced by " $G$ solvable" in the case where $n$ is a prime.

4.3. Hurwitz spaces. Given an integer $r \geq 3$ and a finite group $G$ (resp. a subgroup $G \subset S_{n}$ ), there is a coarse moduli space called a Hurwitz space for Gcovers of $\mathbb{P}^{1}$ of group $G$ (resp. for mere covers of $\mathbb{P}^{1}$ of degree $n$ and geometric monodromy group $G \subset S_{n}$ ) with $r$ branch points. We view it here as a (reducible) variety defined over $\mathbb{Q}$; it can be more generally defined as a scheme over some extension ring of $\mathbb{Z}[1 /|G|]$. We do not distinguish between the G-cover and mere cover situations and use the same notation $\mathrm{H}_{r}(G)$ for the Hurwitz space.

A central moduli property is that for any field $k$ of characteristic 0 , there is a one-to-one correspondence between the set of $\bar{k}$-rational points on $\mathrm{H}_{r}(G)$ and the set of isomorphisms classes of (G-or mere) covers defined over $\bar{k}$ with the given invariants. Furthermore, for every closed point $[f] \in \mathrm{H}_{r}(G)$, the field $k([f])$ is the field of moduli of the corresponding (G- or mere) cover $f$. We refer to [DD97] for more on fields of moduli; in standard situations (e.g. $Z(G)=\{1\}$ for G-covers, $\operatorname{Cen}_{S_{n}}(G)=\{1\}$ for mere covers) and in most situations below, the field of moduli is a field of definition of $f$ and is the smallest one.

Denote by $\mathrm{U}_{r}$ the configuration space for finite subsets of $\mathbb{P}^{1}$ of cardinality $r$. The map $\Psi_{r}: \mathrm{H}_{r}(G) \rightarrow \mathrm{U}_{r}$ that sends each isomorphism class of cover $[f]$ in $\mathrm{H}_{r}(G)$ to its branch point set $\mathbf{t} \in \mathrm{U}_{r}$ is an étale cover defined over $\mathbb{Q}$. The geometrically irreducible components of $\mathrm{H}_{r}(G)$ correspond to the connected components of $\mathrm{H}_{r}(G) \otimes_{\mathbb{Q}} \mathbb{C}$, which in turn correspond to the orbits of the so-called Hurwitz monodromy action of the fundamental group of $\bigcup_{r}$ (the Hurwitz group $\mathcal{H}_{r}$ ) on a fiber $\Psi_{r}^{-1}(\mathbf{t})\left(\mathbf{t} \in \mathrm{U}_{r}(\bar{k})\right)$. For more on Hurwitz spaces, see Völ96] or [Dèb99a].

The variety $U_{r}$ is a Zariski open subset of the projective space $\mathbb{P}^{r}$. For any given component $\mathrm{H}$ of $\mathrm{H}_{r}(G)$, normalizing $\mathbb{P}^{r}$ in the function field $\overline{\mathbb{Q}}(\mathrm{H})$ provides a $\overline{\mathbb{Q}}$-mere cover $\left(\Psi_{r}\right)_{\overline{\mathrm{H}}}: \overline{\mathrm{H}} \rightarrow \mathbb{P}^{r}$. We will apply our specialization results to this mere cover. 
Assume that $\left(\Psi_{r}\right)_{\overline{\mathrm{H}}}: \overline{\mathrm{H}} \rightarrow \mathbb{P}^{r}$ is defined over a field $k$. For $\mathbf{t}_{0} \in \mathrm{U}_{r}(k)$, consider the specialization algebra $\prod_{l=1}^{s} k(\mathrm{H})_{\mathbf{t}_{0}, l} / k$ of $\left(\Psi_{r}\right)_{\overline{\mathrm{H}}}$ at $\mathbf{t}_{0}$. The fields $k(\mathrm{H})_{\mathbf{t}_{0}, l}(l=$ $1, \ldots, s)$ are the fields of moduli of all the $\bar{k}$-covers $\left[f: X \rightarrow \mathbb{P}^{1}\right]$ in $\mathrm{H}$ with branch divisor $\mathbf{t}_{0}$.

Definition 4.7. The $k$-étale algebra $\prod_{l=1}^{s} k(\mathrm{H})_{\mathbf{t}_{0}, l} / k$ is called the $k$-algebra of fields of moduli (or of smallest fields of definition when fields of moduli are fields of definition) of the $\bar{k}$-covers (mere or G-) $f: X \rightarrow \mathbb{P}^{1}$ in $\mathrm{H}$ with branch divisor $\mathbf{t}_{0}$.

In this situation we have the following result. In (b) (ii), where $k$ is a number field and $v$ is a place of $k$, we use the notation $k_{v}^{\mathrm{ur}, f}(f \in \mathbb{N}, f>0)$ for the unramified extension of $k_{v}$ of degree $f$.

Corollary 4.8. Let $k$ be a field and $\mathrm{H}$ be a component of $\mathrm{H}_{r}(G)$ such that $\left(\Psi_{r}\right)_{\overline{\mathrm{H}}}$ : $\overline{\mathrm{H}} \rightarrow \mathbb{P}^{r}$ is a k-mere cover of geometric monodromy group $S_{N}$ with $N=\operatorname{deg}\left(\left(\Psi_{r}\right)_{\overline{\mathrm{H}}}\right)$.

(a) If $k$ is PAC of characteristic 0 and $\prod_{l=1}^{s} E_{l} / k$ a $k$-étale algebra with $\sum_{l=1}^{s}\left[E_{l}\right.$ : $k]=N$, there exists a Zariski-dense subset $\mathcal{U} \subset \cup_{r}(k)$ such that for each $\mathbf{t}_{0} \in \mathcal{U}$, the $k$-étale algebra $\prod_{l=1}^{s} E_{l} / k$ is the $k$-algebra of smallest fields of definition of the $\bar{k}$-covers (mere or $G$-) $f: X \rightarrow \mathbb{P}^{1}$ in $\mathbf{H}$ with branch divisor $\mathbf{t}_{0}$.

(b) There exist two constants $p(r, G)$ and $q(r, G)$ depending only on the integer $r \geq 3$ and the group $G$ with the following property. Let $k$ be a number field, $\mathcal{S}$ be a finite subset of finite places of $k$ with residue field of order $\geq q(r, G)$ and residue characteristic $>p(r, G)$, and for each $v \in \mathcal{S}$, let $d_{v, 1}, \ldots, d_{v, s_{v}}$ be positive integers with $\sum_{l=1}^{s_{v}} d_{v, l}=N$. Then there is a Zariski-dense subset $\mathcal{U} \subset \cup_{r}(k)$, of the form $\mathcal{U}=\cup_{r}(k) \cap \prod_{v \in \mathcal{S}} U_{v}$ for some $v$-adic open subsets $U_{v} \subset \mathrm{U}_{r}\left(k_{v}\right)$, such that for each $\mathbf{t}_{0} \in \mathcal{U}$,

(i) the field of moduli of each of the $\bar{k}$-covers $f: X \rightarrow \mathbb{P}^{1}$ in $\mathrm{H}$ with branch divisor $\mathbf{t}_{0}$ is a degree $N$ extension of $k$, and,

(ii) for each $v \in \mathcal{S}$, the $k_{v}$-algebra of smallest fields of definition of the (mere or G-) $\overline{k_{v}}$-covers $f \otimes_{\bar{k}} \overline{k_{v}}$ (for any given embedding $\bar{k} \hookrightarrow \overline{k_{v}}$ ) in $\mathrm{H}$ with branch divisor $\mathbf{t}_{0}$ is the $k_{v}$-étale algebra $\prod_{l=1}^{s_{v}} k_{v}^{\mathrm{ur}, d_{v, l}} / k_{v}$.

Proof. Statement (a) is a straightforward application of Corollary 3.1, applied to the $k$-mere cover $\left(\Psi_{r}\right)_{\overline{\mathrm{H}}}$ and combined with Definition 4.7 and the fact that over a PAC field, the field of moduli is always a field of definition [DD97.

For statement (b), we apply Corollary 3.5 to the $k$-mere cover $\left(\Psi_{r}\right)_{\overline{\mathrm{H}}}$ with $\mathcal{B}=\mathbb{P}^{r}$ and to the $k_{v}$-étale algebras $\prod_{l=1}^{s_{v}} k_{v}^{\mathrm{ur}, d_{v, l}} / k_{v}(v \in \mathcal{S})$. The geometric monodromy group of $\left(\Psi_{r}\right)_{\overline{\mathrm{H}}}$ being $S_{N}$, the first assumption holds. The second one holds too (for any finite set $\mathcal{S}$ of finite places), as $\mathbb{P}^{r}$ is a $k$-rational variety. The branch locus $D=\mathbb{P}^{r} \backslash \mathrm{U}_{r}$ consists of hyperplane sections that cross normally over $k$. Only finitely many places of $k$ may not satisfy condition (good-red) from Corollary [3.3. Take for $p(r, G)$ the largest characteristic of these exceptional places and for $q(r, G)$ the constant $C\left(\left(\Psi_{r}\right)_{\overline{\mathrm{H}}}, \mathbb{P}^{r}\right)$ from Addendum 3.5. These constants can indeed be chosen depending on $r$ and $G$, and not on the number field $k$ (Remark 3.4). Assuming $p_{v}>p(r, G)$ and $q_{v} \geq q(r, G)(v \in \mathcal{S})$ then guarantees that the third assumption of Corollary 3.5 holds. Statement (b)(ii) then corresponds to the conclusion of Corollary 3.5, combined with Definition 4.7 and the fact that, as a consequence of (good-red), the field of moduli of each cover $f \otimes_{\bar{k}} \overline{k_{v}}$ is a field of definition [DH98]. 
In order to obtain (b)(i), one uses the standard trick recalled in 4.1 .1 adding to $\mathcal{S}$ well-chosen places $v$ with corresponding $k_{v}$-étale algebras and applying Corollary 3.5 to this larger set of places assures that the $k$-specialization algebra of $\left(\Psi_{r}\right)_{\overline{\mathrm{H}}}$ at $\mathbf{t}_{0}$ is a single field extension $k(\mathrm{H})_{\mathbf{t}_{0}} / k$ of degree $N$.

There is in Corollary 4.8 the assumption that $\left(\Psi_{r}\right)_{\overline{\mathrm{H}}}: \overline{\mathrm{H}} \rightarrow \mathbb{P}^{r}$ be a $k$-mere cover of geometric monodromy group $S_{N}$. This assumption can be checked in practical situations. Indeed, the geometric monodromy group is the image group of the Hurwitz monodromy action (restricted to the component $\mathrm{H}$ ), which can be made totally explicit.

\section{REFERENCES}

[Ang07] Julien Angeli, Trinômes irréductibles résolubles sur un corps de nombres, Acta Arith. 127 (2007), no. 2, 169-178. MR2289982(2007m:11155)

[Ang09] Trinômes à petits groupes de Galois, Thèse de doctorat, Université de Limoges, 2009.

[Bec91] Sybilla Beckmann, On extensions of number fields obtained by specializing branched coverings, J. Reine Angew. Math. 419 (1991), 27-53. MR1116916 (93a:11095)

[BS09] Lior Bary-Soroker, Dirichlet's theorem for polynomial rings, Proc. Amer. Math. Soc. 137 (2009), 73-83. MR2439427 (2009h:12007)

[BS10] , Irreducible values of polynomials, Adv. Math. 229 (2012), no. 2, 854-874. MR2855080 (2012j:11184)

[Coh80] Stephan D. Cohen, The Galois group of a polynomial with two indeterminate coefficients, Pacific J. Math. 90 (1980), no. 1, 63-76. MR599320 (83j:12020a)

[Coh81] , Corrections to [Coh80], Pacific J. Math. 97 (1981), no. 2, 483-486. MR641176 (83j:12020b)

[DD97] Pierre Dèbes and Jean-Claude Douai, Algebraic covers: Field of moduli versus field of definition, Annales Sci. E.N.S. 30 (1997), 303-338. MR.1443489 (98k:11081)

[Dèb99a] Pierre Dèbes, Arithmétique et espaces de modules de revêtements, Number Theory in Progress, (K. Gyory, H. Iwaniec and J. Urbanowicz, eds.), Walter de Gruyter, 1999, pp. 75-102. MR1689500 (2000c:14029)

[Dèb99b] _ Density results for Hilbert subsets, Indian J. Pure and Applied Math. 30 (1999), no. 1, 109-127. MR1677959(2000c:12004)

[Dèb99c] _ Galois covers with prescribed fibers: The Beckmann-Black problem, Ann. Scuola Norm. Sup. Pisa, Cl. Sci. (4) 28 (1999), 273-286. MR1736229 (2000m:12006)

[Dèb09] _ Arithmétique des revêtements de la droite, at http://math.univlille1.fr/ pde/ens.html.

[DG11] Pierre Dèbes and Nour Ghazi, Galois covers and the Hilbert-Grunwald property, Ann. Inst. Fourier 62 (2012), no. 3, 989-1013. MR3013814

[DH98] Pierre Dèbes and David Harbater, Fields of definition of p-adic covers, J. Reine Angew. Math. 498 (1998), 223-236. MR.1629870 (99e:12006)

[DL] Pierre Dèbes and François Legrand, Twisted covers and specializations, GaloisTeichmueller theory and Arithmetic Geometry, Proceedings for Conferences in Kyoto (October 2010), H. Nakamura, F. Pop, L. Schneps, A. Tamagawa eds., Advanced Studies in Pure Mathematics 63, 2012, pages 141-163.

[DW08] Pierre Dèbes and Yann Walkowiak, Bounds for Hilbert's irreducibility theorem, Pure \& Applied Math. Quarterly 4/4 (2008), 1059-1083. MR2441693 (2010e:12003)

[FJ04] Michael D. Fried and Moshe Jarden, Field arithmetic, Ergebnisse der Mathematik und ihrer Grenzgebiete, vol. 11, Springer-Verlag, Berlin, 2004 (first edition 1986). MR.868860 (89b:12010)

[Sch00] Andrzej Schinzel, Polynomials with special regard to reducibility, Encyclopedia of Mathematics and its Applications, vol. 77, Cambridge University Press, 2000. MR.1770638 (2001h:11135)

[Ser92] Jean-Pierre Serre, Topics in Galois theory, Research Notes in Mathematics, Jones and Bartlett Publishers, 1992. MR:1162313 (94d:12006) 
[Uch70] Koji Uchida, Galois group of an equation $x^{n}-a x+b=0$, Tohoku Math. Journ. 22 (1970), 670-678. MR0277505 (43:3238)

[Vö196] Helmut Völklein, Groups as Galois groups, Cambridge Studies in Advanced Mathematics, vol. 53, Cambridge University Press, 1996. MR,1405612 (98b:12003)

Laboratoire Paul Painlevé, Mathématiques, Université Lille 1, 59655 Villeneuve D'Ascq Cedex, France

E-mail address: Pierre.Debes@math.univ-lille1.fr

Laboratoire Paul Painlevé, Mathématiques, Université Lille 1, 59655 Villeneuve D'Ascq Cedex, France

E-mail address: Francois.Legrand@math.univ-lille1.fr 